\title{
Notificación de muerte en urgencias médicas: el rol educativo del actor profesional
}

\author{
Edgar Landa-Ramírez ${ }^{1 *}$ y Antonio López-Gómez ${ }^{2 *}$ \\ ${ }^{1}$ Programa de Psicología Urgencias/División de Urgencias y Observación, Hospital General «Dr. Manuel Gea González»; Programa de Psicología \\ de la Salud y Medicina Conductual, Facultad de Psicología, Universidad Nacional Autónoma de México; ${ }^{2}$ División de Urgencias y Observación, \\ Hospital General «Dr. Manuel Gea González. Ciudad de México, México
}

La notificación de muerte es una situación frecuente en el Área de Urgencias, y los médicos suelen reconocer que se trata de una de las actividades más estresantes, incómodas y para la que menos preparados se perciben ${ }^{1}$. No obstante, dar malas noticias es una habilidad susceptible de ser adquirida y mejorada por medio de la educación, el entrenamiento continuo y la práctica. En los últimos años, se ha fomentado el uso de distintos modelos (por ejemplo, SPIKES y GRIEV ING) $)^{2,3}$ que entrenan al personal de urgencias para dar la notificación de muerte de manera humana y empática. Los programas educativos suelen considerar el juego de roles como un elemento central para el desarrollo de las habilidades. Durante el juego de roles, los estudios involucran a las siguientes personas para practicar la notificación de muerte: actores profesionales, voluntarios, médicos que juegan el rol del familiar y familiares del paciente fallecido. Con base en nuestra experiencia, creemos que es mucho más benéfico incluir a actores profesionales para el entrenamiento en la notificación de muerte, ya que hemos notado que, a pesar del alto compromiso de los médicos y voluntarios con sus personajes durante el juego de roles, existen casos cuya complejidad emocional extrema no les permite representar correctamente la respuesta del familiar en la notificación de muerte.

Aunque consideramos invaluable la participación de familiares de pacientes fallecidos, no dejamos de preguntarnos sobre los posibles efectos psicológicos negativos que podrían experimentar al rememorar al ser querido que falleció. Por tal razón, creemos indispensable incluir en el entrenamiento de malas noticas a actores profesionales que sean capaces de transmitir los déficits en las respuestas comportamentales, cognitivas y emocionales cercanas a las que el familiar vive cuando recibe la noticia y con las cuales van a lidiar los médicos el resto de su vida profesional ${ }^{4}$.

En el siguiente video, creado en nuestro equipo de trabajo, en la División de Urgencias Médicas del Hospital General «Dr. Manuel Gea González» (https://youtu. be/1bn3o794KtE), se puede apreciar la diferencia e impacto, cuando interpreta el mismo caso un médico adscrito al Servicio de Urgencias y una actriz profesional con cinco años de experiencia representando casos en el área de la salud. Durante el tiempo en que hemos impartido el programa educativo GRIEV_ING en notificación de muerte en nuestro hospital, los residentes han comentado que el uso de la actriz es mucho más real,

\footnotetext{
Correspondencia:

*Edgar Landa-Ramírez

Av. Universidad, 3114

Col. Copilco Universidad, Del. Coyoacán Fecha de recepción: 06-08-2018

Disponible en internet: 08-02-2019

C.P. 04510, Ciudad de México, México

Fecha de aceptación: 12-12-2018

Rev Educ Investig Emer. 2019;1(1):8-9

E-mail: edgar_landa_ramirez@yahoo.com.mx

DOI: 10.24875/REIE.M19000005

www.medicinadeemergencias.com

2604-6520 C 2019 Sociedad Mexicana de Medicina de Emergencia, AC. Publicado por Permanyer México SA de CV. Este es un artículo Open Access bajo la licencia CC BY-NC-ND (http://creativecommons.org/licenses/by-nc-nd/4.0/).
} 
más cercano a lo que viven diariamente, y lo consideran muy útil en su formación profesional. Dada nuestra experiencia y la creciente evidencia científica sobre cómo comunicar malas noticias, consideramos indispensable incluir a actores profesionales en este tipo de entrenamientos educativos para colaborar en el desarrollo de las habilidades necesarias para dar la notificación de muerte en el Departamento de Urgencias.

\section{Bibliografía}

1. Shoenberger JM, Yeghiazarian S, Rios C, Henderson SO. Death notification in the emergency department: survivors and physicians. West $J$ Emerg Med. 2013;14(2):181-5.

2. Park I, Gupta A, Mandani K, Haubner L, Peckler B. Breaking bad news education for emergency medicine residents: A novel training module using simulation with the SPIKES protocol. J Emerg Trauma Shock. 2010; 3(4):385-8.

3. Hobgood C, Harward D, Newton K, Davis W. The educational intervention "GRIEV_ING» improves the death notification skills of residents. Acad Emerg Med. 2005;12(4):296-301.

4. Cole EA. My Life as a (Fake) Patient. N Engl J Med. 2015;373(24):2302-3. 